\title{
Large tunable anomalous Hall effect in the kagome antiferromagnet $U_{3} \mathbf{R u}_{4} \mathbf{A l}_{12}$
}

\author{
T. Asaba $\odot,{ }^{1, *}$ Ying Su, ${ }^{2}$ M. Janoschek,${ }^{1,3}$ J. D. Thompson, ${ }^{1}$ S. M. Thomas, ${ }^{1}$ E. D. Bauer, ${ }^{1}$ Shi-Zeng Lin $\odot,{ }^{2}$ and F. Ronning ${ }^{4, \dagger}$ \\ ${ }^{1}$ MPA-Q, Los Alamos National Laboratory, New Mexico 87545, USA \\ ${ }^{2}$ Theoretical Division, T-4 and CNLS, Los Alamos National Laboratory, New Mexico 87545, USA \\ ${ }^{3}$ Laboratory for Neutron and Muon Instrumentation, Paul Scherrer Institute, CH-5232 Villigen, Switzerland \\ ${ }^{4}$ Institute for Materials Science, Los Alamos National Laboratory, New Mexico, 87545, USA
}

(Received 17 July 2019; revised 13 January 2020; accepted 11 June 2020; published 16 July 2020)

\begin{abstract}
The Berry curvature in magnetic systems is attracting interest due to the potential tunability of topological features via the magnetic structure. $f$ electrons, with their large spin-orbit coupling, abundance of noncollinear magnetic structures, and high electronic tunability, are attractive candidates to search for tunable topological properties. In this study, we measure anomalous Hall effect (AHE) in the distorted kagome heavy fermion antiferromagnet $\mathrm{U}_{3} \mathrm{Ru}_{4} \mathrm{Al}_{12}$. A large intrinsic $\mathrm{AHE}$ in high fields reveals the presence of a large Berry curvature. Moreover, the fields required to obtain the large Berry curvature are significantly different between $B \| a$ and $B \| a^{*}$, providing a mechanism to control the topological response in this system. Theoretical calculations illustrate that this sensitivity may be due to the heavy fermion character of the electronic structure. These results shed light on the Berry curvature of a strongly correlated band structure in magnetically frustrated heavy fermion materials but also emphasize $5 f$ electrons as an ideal playground for studying field-tuned topological states.
\end{abstract}

DOI: 10.1103/PhysRevB.102.035127

\section{INTRODUCTION}

The Berry curvature of a material is a property of the electronic structure that provides an anomalous transverse velocity to electrons traveling in a solid. For insulators, the integral of the Berry curvature becomes quantized leading to the notion that an electronic structure has a topology defined by its Berry curvature. This topology can lead to dramatic observables such as quantized conductance and novel boundary states [1-5]. By tuning the topology of a system one may hope to control these properties. This has been demonstrated in several noncollinear antiferromagnets (AFM) and ferromagnets (FM), where the opposite Berry curvature from two different domains can be accessed by flipping a small applied magnetic field [6-10]. The reversal of the Berry curvature is witnessed by the change in sign of a large intrinsic component to the anomalous Hall effect [11]. A finite Berry curvature is a consequence of spin-orbit coupling (SOC). SOC enables a magnetic field and/or magnetic structure to modify the electronic structure [12-14]. This demonstrates an additional mechanism to tune the topological response of a system with a rotating magnetic field, but for typical electronic energy scales, one would expect a field of a few Tesla to be a weak perturbation.

Strong electronic correlations can further broaden the landscape of topological materials, for instance, by creating novel fractionalized particles [15-18]. Importantly, it can amplify the tunability of materials through increased susceptibility to external perturbations. $f$-electron heavy fermion systems are ideal for these types of studies, as the renormalized

\footnotetext{
*tasaba@lanl.gov

${ }^{\dagger}$ fronning@lanl.gov
}

electronic energy scales are 100-1000 times smaller than ordinary metals. Furthermore, strong SOC can lead to topologically nontrivial properties as well as noncollinear spin structures [19-21].

Here we report Hall effect measurements on the heavy fermion, noncollinear antiferromagnet $\mathrm{U}_{3} \mathrm{Ru}_{4} \mathrm{Al}_{12}$. With an applied magnetic field we find a significant nonlinear anomalous Hall response, which can be tuned by small rotations of the magnetic field. Theoretical calculations reveal that this can be understood as a consequence of the magnetic field exceeding the linear response regime due to a small electronic energy scale of heavy fermion quasiparticles. Hence, the electronic structure, and consequently the Berry curvature, is significantly modified by the field strength and orientation. This work demonstrates that $5 f$-based materials are interesting model systems to investigate the tunability of the Berry curvature in the presence of strong electronic correlations.

\section{EXPERIMENTS}

The heavy-fermion antiferromagnet $\mathrm{U}_{3} \mathrm{Ru}_{4} \mathrm{Al}_{12}$ has a $\mathrm{Gd}_{3} \mathrm{Ru}_{4} \mathrm{Al}_{12}$ type hexagonal crystal structure. Distorted kagome nets of uranium atoms govern the magnetism. Due to the $5 f$ electrons of uranium and the frustrated kagome geometry, the system orders at $8 \mathrm{~K}$ in a unique noncollinear magnetic structure shown in Fig. 1(a) [22]. Neutron scattering measurements show that the spins are rotated \pm 60 degrees in each triangle, resulting in a net ferromagnetic component in-plane [23]. This ferromagnetic component is then canceled out by adjacent layers that have the opposite spin arrangement, forming the antiferromagnetic structure. The system possesses a large Sommerfeld coefficient $\gamma$ of $110 \mathrm{~mJ} / \mathrm{mol}-\mathrm{U}$ $\mathrm{K}^{2}$ suggesting a large effective mass within the magnetically ordered state [see Fig. 1(b)]. 

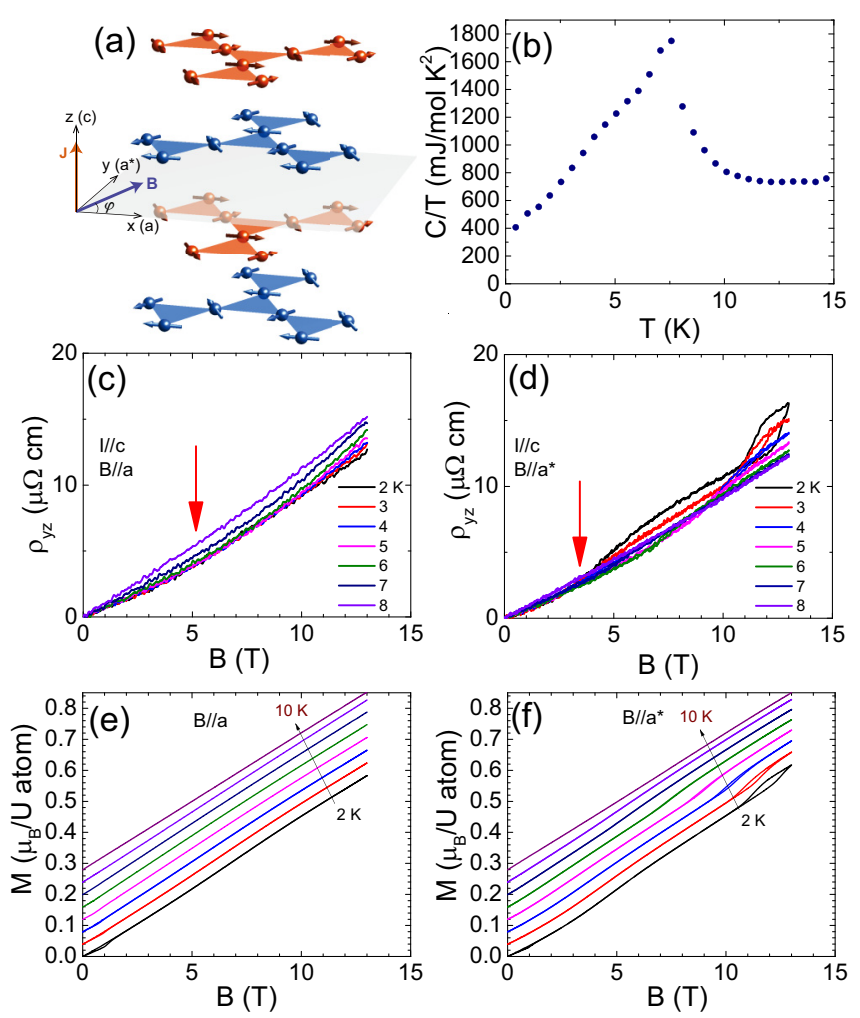

FIG. 1. Magnetization and anomalous Hall resistivity at different temperatures. (a) The magnetic structure of $\mathrm{U}_{3} \mathrm{Ru}_{4} \mathrm{Al}_{12}$. Only uranium atoms are shown. The arrows indicate the magnetic structure determined by neutron scattering measurements [23]. (b) Specific heat data of $\mathrm{U}_{3} \mathrm{Ru}_{4} \mathrm{Al}_{12}$. (c),(d) Anomalous Hall resistivity with $J \| \mathrm{c}$ and (c) $B \| a$ and (d) $B \| a^{*}$. (e),(f) In-plane magnetic field dependence of magnetization with (e) $B \| a$ and (f) $B \| a^{*}$. The curves are offset by $0.04 \mu_{B} / \mathrm{U}$ atom for clarity.

Our main observation is a surprising nonlinearity and angular dependence in the transverse resistivity (anomalous Hall effect, AHE) in comparison to the magnetization as shown in Fig. 1(c) and Fig. 1(d) for $B \| a$ and $B \| a^{*}$, respectively. At low fields, $\rho_{y z}$ and $M$ increase linearly with applied fields. However, an unexpected field-induced enhancement of $\rho_{y z}$ is observed in both orientations at low temperatures and low fields $(<10 \mathrm{~T})$, as indicated by arrows.

Figures 1(e) $(B \| a)$ and 1(f) $\left(B \| a^{*}\right)$ show the magnetic field dependence of magnetization at different temperatures and different field orientations. In both orientations, at low fields hysteresis loops are observed, illustrating antiferromagnetic domain reorientation (see SM). Also, the susceptibility is almost identical in $a$ and $a^{*}$ at low fields, indicating an isotropic response. With increasing field, both orientations show a nearly linear field dependence. An additional jump with a hysteresis loop is observed in the $B \| a^{*}$ orientation at the critical field $B_{M}=12 \mathrm{~T}$ at $T=2 \mathrm{~K}$, which is also seen in magnetoresistance (MR) data shown in the Supplemental Material and in magnetization and ultrasound data [24,25]. Thus, it is confirmed that a field-induced phase transition originates from a metamagnetic transition at $B_{M}$. A similar inplane metamagnetic phase-transition anisotropy was observed in $\mathrm{Dy}_{3} \mathrm{Ru}_{4} \mathrm{Al}_{12}$ [26] and $\mathrm{Ho}_{3} \mathrm{Ru}_{4} \mathrm{Al}_{12}$ [27], indicating that this property is related to the crystal electric field anisotropy and the crystal structure.

In contrast to the high-field metamagnetic transition, the field-induced nonlinear anomalous Hall conductivity (AHC) behavior is not due to a phase transition. Indeed, no anomaly is observed in magnetization or specific heat data at the field where $\rho_{y z}$ is suddenly enhanced, as shown in Figs. 1(c) and 1(d). Below, we discuss that the AHC possesses both extrinsic and intrinsic anomalous Hall contributions, and by subtracting a contribution proportional to the magnetization, we find a significant field and temperature dependent intrinsic AHE contribution.

In general, in magnetic materials, the transverse resistivity $\rho_{y z}$ is expressed as

$$
\rho_{y z}=R_{H} B+\mu_{0} R_{s}^{\mathrm{ext}} M+R_{\mathrm{AHE}}^{\mathrm{int}},
$$

where $R_{H}, R_{s}^{\text {ext }}$, and $R_{\mathrm{AHE}}^{\text {int }}$ are ordinary, extrinsic anomalous, and intrinsic anomalous Hall contributions, respectively. The ordinary Hall contribution $R_{H} B$ can be determined at high temperatures and is found to be negligible [24]. Thus, the expression of $\rho_{y z}$ reduces to

$$
\rho_{y z}=\mu_{0} R_{s}^{\mathrm{ext}} M+R_{\mathrm{AHE}}^{\mathrm{int}} .
$$

The extrinsic AHE $\mu_{0} R_{s}^{\text {ext }} M$ originates from a scattering mechanism. The intrinsic AHE $R_{\mathrm{AHE}}^{\text {int }}$ originates from the Berry curvature. Because the magnetic structure shown in Fig. 1(a) is symmetric with the product of the time reversal operation and the inversion symmetry operation $\mathcal{P} \times \mathcal{T}$, $R_{\mathrm{AHE}}^{\text {int }}$ vanishes at zero field. In low fields when $\rho_{y z}$ and $M$ are proportional we cannot distinguish between the intrinsic and extrinsic responses. However, we note that Onoda et al. suggest that the AHC is dominated by the intrinsic component when $\rho>10-100 \mu \Omega \mathrm{cm}$ for rare earth compounds [28]. Since the resistivity of $\mathrm{U}_{3} \mathrm{Ru}_{4} \mathrm{Al}_{12}$ is larger than $300 \mu \Omega \mathrm{cm}$, the anomalous Hall effect could be solely attributed to the intrinsic contribution. If this is the case, the intrinsic AHC would reach about 100 (280) $\Omega^{-1} \mathrm{~cm}^{-1}$ with $\mathrm{B} \| a^{*}(\mathrm{~B} \| c)$ (for B $\| c$ data, see SM [24]).

After subtracting the linear-in- $M$ component, the remaining nonlinear intrinsic AHC at different temperatures is shown in Fig. 2(b). The AHC with different orientations is shown in (c). When $B \| a$ the intrinsic nonlinear anomalous Hall conductivity becomes finite above $B_{a} \simeq 4-5 \mathrm{~T}$. When $B \| a^{*}$ the nonlinear AHC emerges above $B_{a^{*}} \simeq 2-3 \mathrm{~T}$, and the AHC $\Delta \sigma_{y z}^{\text {int }}=-\frac{\Delta \rho_{y z}^{\text {int }}}{\rho_{y y} \rho_{z z}+\rho_{y z}^{2}}$ reaches about $34 \Omega^{-1} \mathrm{~cm}^{-1}$ at $13 \mathrm{~T}$, comparable to ferromagnetic materials.

Given the nonlinear Berry curvature with field magnitude, we also explore the angle dependence of the AHE. The fact that the onset field for the nonlinear AHC is almost double for $B \| a$ than for $B \| a^{*}$ indicates the Berry curvature can be sensitively tuned by rotating the magnetic field. Indeed, the intrinsic nonlinear AHC as a function of tilt angle $\phi$, shown in Fig. 2(d), is remarkably sensitive to the sample orientation. An overall twofold oscillation is expected due to the reorientation of the magnetic structure with the applied magnetic field However, at $B=5 \mathrm{~T}$, the absolute value of $\mathrm{AHC}$ reaches local maxima when $B \| a^{*}$ (namely, 30, 90, 150, 210, 270, and 330 degrees). On the other hand, the absolute value of $\Delta \sigma_{\mathrm{AHE}}^{\text {int }}$ almost vanishes at every 60 degrees, when $B \| a$. This 

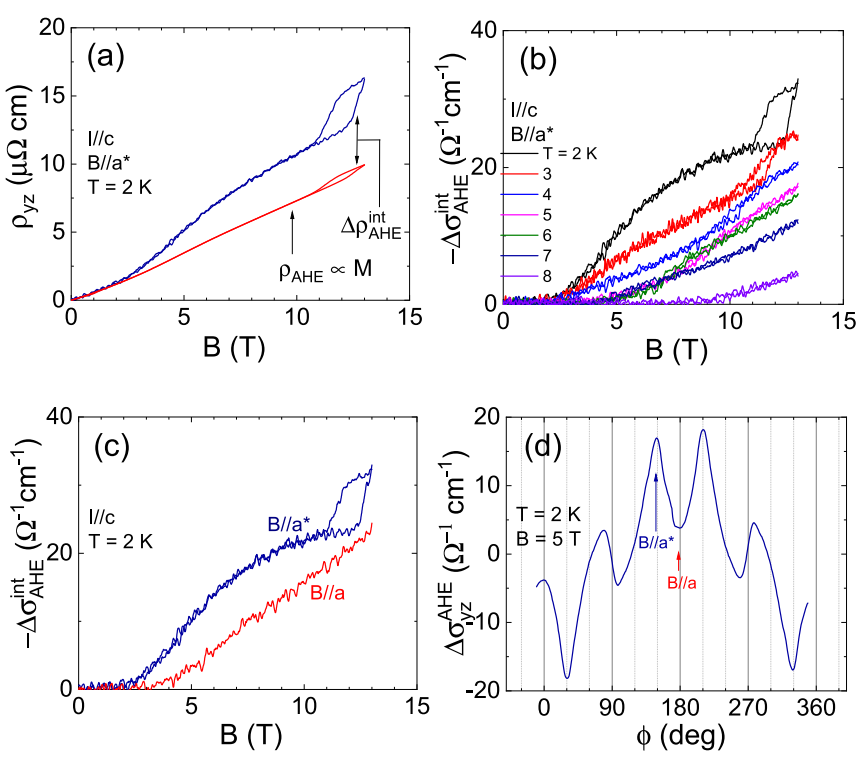

FIG. 2. Intrinsic anomalous Hall conductivity and its angular dependence. (a) Linear and nonlinear contribution of anomalous Hall resistivity with $B \| a^{*}$. The blue (red) curves indicate the total (linear in $M$ ) anomalous Hall resistivity. The difference between blue and red curves represents the nonlinear intrinsic contribution. (b) The temperature dependence of the intrinsic anomalous Hall conductivity after subtracting the extrinsic contribution with $B \| a^{*}$. (c) The intrinsic anomalous Hall conductivity with $B \| a$ and $B \| a^{*}$ at $T=2 \mathrm{~K}$. (d) In-plane angular dependence of nonlinear anomalous Hall conductivity at $B=5 \mathrm{~T}$. $\phi=60 \mathrm{n}$ for $B \| a$ and $\phi=60 \mathrm{n}+30$ for $B \| a^{*}$. The current was applied parallel to the $c$ axis.

demonstrates that the Berry curvature of our system can be highly tuned by small sample rotations.

\section{THEORETICAL MODEL}

To better understand the $\mathrm{AHC}$ in $\mathrm{U}_{3} \mathrm{Ru}_{4} \mathrm{Al}_{12}$ with field and angle we construct a minimal model that captures the essential physics (see SM). The coplanar spin structure suggests that the intrinsic contribution to the AHE is a consequence of the Berry curvature created by the electronic structure in momentum space, as opposed to a real space contribution $[29,30]$. We introduce a Kondo lattice model to describe our system (see SM)

$$
\begin{aligned}
H= & \sum_{\langle i \alpha, j \beta\rangle} t_{i \alpha, j \beta} c_{i \alpha}^{\dagger} c_{j \beta}-J \sum_{i \alpha} c_{i \alpha}^{\dagger} \boldsymbol{S}_{i \alpha} \cdot \boldsymbol{\sigma} c_{i \alpha} \\
& -\sum_{i \alpha} c_{i \alpha}^{\dagger} \boldsymbol{B} \cdot \boldsymbol{\sigma} c_{i \alpha}+i t_{i \alpha, j \beta}^{s o} \sum_{\langle i \alpha, j \beta\rangle} c_{i \alpha}^{\dagger} \boldsymbol{n}_{\alpha \beta} \cdot \sigma c_{j \beta},
\end{aligned}
$$

where $c_{i \alpha}=\left(c_{i \alpha \uparrow}, c_{i \alpha \downarrow}\right)^{\top}$ is the conduction electron annihilation operator of a two-component spinor at the $i$ th unit cell and of the $\alpha$ sublattice. The localized $f$ electrons are responsible for the magnetic moments $S_{i}$, which are treated classically. We take the experimentally measured spin configuration $\boldsymbol{S}_{i}$ [23]. The spin-orbit coupling (SOC) vectors $\boldsymbol{n}_{\alpha \beta}$ respect the $\mathcal{T}$ and $\mathcal{P}$ symmetries and are defined in SM [24]. As discussed in detail in the Supplemental Material [24], we need to break $\mathcal{P} \mathcal{T},\left\{\mathcal{P C}_{2 z} \mid(0,0,1 / 2)\right\}$, and $\left\{\mathcal{T} \mathcal{M}_{z} \mid(0,0,1 / 2)\right\}$ symmetries to have a nonzero AHC. Here
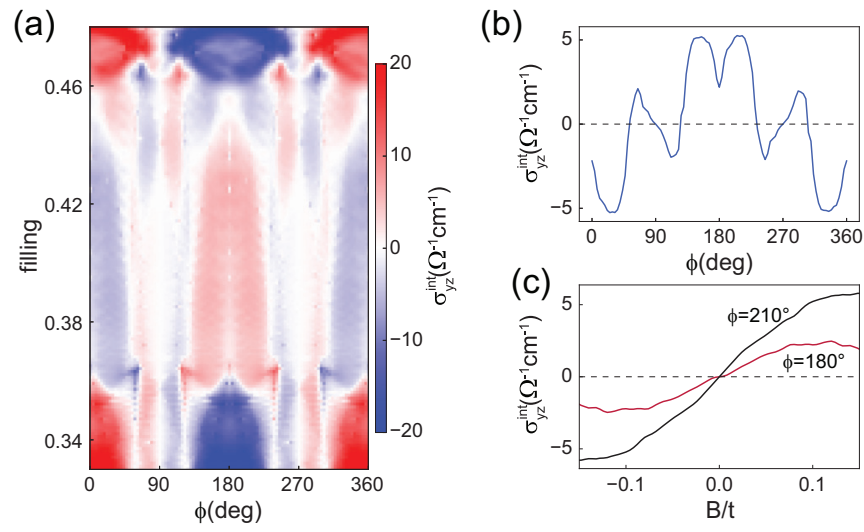

FIG. 3. Theoretically calculated intrinsic anomalous Hall conductance in model Eq. (3). (a) The anomalous Hall conductance $\sigma_{y z}^{\text {int }}$ as a function of the field angle $\phi$ and electron filling. (b) $\sigma_{y z}^{\text {int }}$ vs $\phi$ for fixed filling at 0.38 . (c) $\sigma_{y z}^{\text {int }}$ as function of the field strength $B$ for the two field angles $\phi=180^{\circ}$ and $210^{\circ}$. Here we use the lattice parameter $a_{1}=0.862 \mathrm{~nm}$ to obtain $\sigma_{y z}^{\text {int }}$.

$\mathcal{C}_{2 z}$ is the twofold rotation of the lattice with respect to the rotation axis along the $z$ direction, $\mathcal{M}_{z}$ denotes the mirror symmetry with the mirror plane perpendicular to the $z$ direction, and $(0,0,1 / 2)$ denotes a nonprimitive translation along the $z$ direction by half a lattice constant. The $\boldsymbol{B}$ field breaks $\mathcal{P} \mathcal{T}$ and the SOC breaks $\left\{\mathcal{P} \mathcal{C}_{2 z} \mid(0,0,1 / 2)\right\}$ and $\left\{\mathcal{T} \mathcal{M}_{z} \mid(0,0,1 / 2)\right\}$ symmetries.

We calculate the intrinsic anomalous Hall conductivity $\sigma_{y z}^{\text {int }}$ as a function of field angle $\phi$ and electron filling, see Fig. 3(a). In a range of filling, from about 0.36 to 0.42 , the theoretically calculated $\sigma_{y z}^{\text {int }}$ is consistent with the experimental results. The field strength and direction dependence of $\sigma_{y z}$ at one typical filling at 0.38 are displayed in Figs. 3(b) and 3(c). The simple model captures semiquantitatively the experimental observation in Figs. 2(d) and 2(c).

\section{DISCUSSION}

In Fig. 4, we show the magnetic phase diagram and nonlinear AHC contour plot of $\mathrm{U}_{3} \mathrm{Ru}_{4} \mathrm{Al}_{12}$. With the magnetic field $B$ applied parallel to $a^{*}$, a magnetic phase transition is observed in MR, magnetization, and heat capacity measurements (see SM), which is consistent with a recent study [25]. At low fields, the system stays in the antiferromagnetic phase I (AF I). At high fields, there is a metamagnetic transition and the system enters antiferromagnetic phase II (AF II). Surprisingly, the AHC behaves almost independently from the magnetic phases. At low temperatures, a magnetic field $B_{a^{*}}$ required to push the Berry curvature to a nonlinear regime is almost constant and much smaller than $B_{M}$. At high temperatures, $B_{a^{*}}$ increases rapidly as the temperature approaches $T_{N}$, while $B_{M}$ decreases. Neutron scattering measurements at high fields would be helpful to understand the magnetic structure of the AF II phase and the evolution of the AHC.

In recent reports, the sign of the Berry curvature was switched by flipping the domain structure [6-10], but it is quite rare to observe an in-plane Berry-curvature switching without inducing a magnetic transition. This is reasonable because in most cases the in-plane magnetic anisotropy is quite 


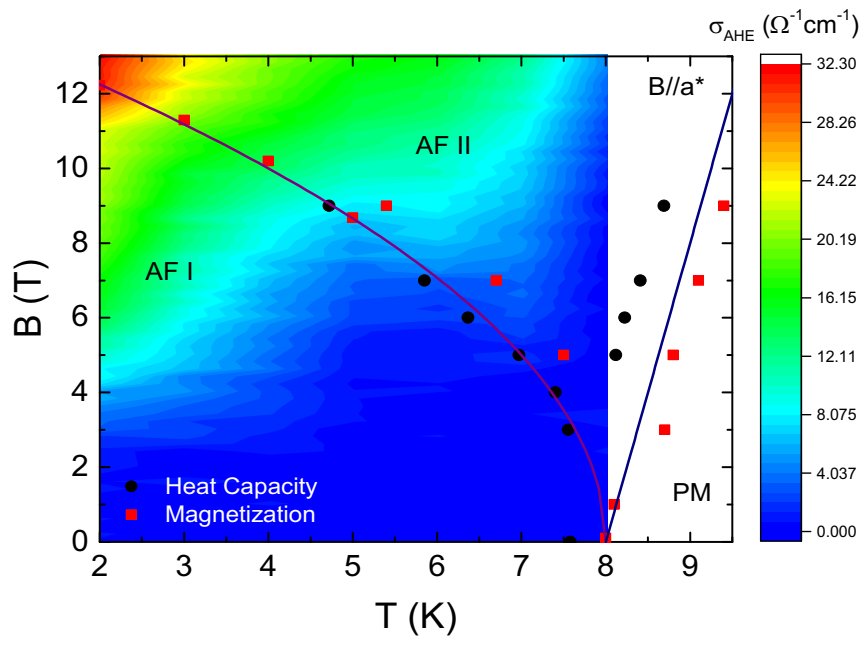

FIG. 4. Magnetic and topological phase diagram of $\mathrm{U}_{3} \mathrm{Ru}_{4} \mathrm{Al}_{12}$. The magnetic field is applied parallel to $a^{*}$. The magnetic phase transitions are determined by heat capacity and magnetization measurements, which are consistent with MR. The contour plot indicates the amplitude of the nonlinear AHC.

small, as is also the case in $\mathrm{U}_{3} \mathrm{Ru}_{4} \mathrm{Al}_{12}$. What causes such an anisotropic and field-dependent behavior of Berry curvature in $\mathrm{U}_{3} \mathrm{Ru}_{4} \mathrm{Al}_{12}$ ? A noncoplanar spin texture could generate such an effect but would require the coplanar spin arrangement in zero field to cant out of the plane. Our theoretical model demonstrates that a field and angular dependent momentumspace Berry curvature could arise in $\mathrm{U}_{3} \mathrm{Ru}_{4} \mathrm{Al}_{12}$ if the strength of the field becomes a sizable fraction of the bandwidth. For most materials this is not possible with today's magnets, but the renormalized bandwidth found in heavy fermion materials enables this mechanism as a result of strong electronic correlations.

\section{CONCLUSIONS}

We have demonstrated that a heavy fermion noncollinear antiferromagnet can be driven into a regime with a nonlinear response of the Berry curvature. A similar field-induced Berry curvature was also observed in the noncollinear antiferromagnet and attributed to the proximity of Weyl nodes to the Fermi energy [14]. The total $\mathrm{AHC}$ in $\mathrm{U}_{3} \mathrm{Ru}_{4} \mathrm{Al}_{12}$ reaches $0.21 \mathrm{e}^{2} / h a$ for $\sigma_{y z}$ where $a$ is the $a$-axis lattice parameter of which a minimum of $0.08 \mathrm{e}^{2} / h a$ can be attributed to an intrinsic Berry curvature effect. For $\sigma_{x y}$, an even larger value of total AHC $\left(0.68 \mathrm{e}^{2} / h c\right)$ was reached. ( $c$ is the $c$-axis lattice parameter). These large values are found despite $\mathrm{U}_{3} \mathrm{Ru}_{4} \mathrm{Al}_{12}$ being a three-dimensional electron system. The combination of large effective masses, crystal electric fields, and a frustrated distorted kagome lattice enables this system to possess strong sensitivity of the Berry curvature to the magnitude and direction of an applied field and sample rotation, further illustrating that $f$-electron systems are fruitful playgrounds to explore tuning of their Berry curvature.

\section{ACKNOWLEDGMENTS}

We thank V. Zapf for assistance with measurements at the National High Magnetic Field Laboratory. The experimental work was supported by the U.S. Department of Energy, Office of Science, Basic Energy Sciences, Materials Sciences and Engineering Division. The theoretical work was carried out under the auspices of the U.S. DOE Award No. DE-AC5206NA25396 through the LDRD program and was supported by the Center for Nonlinear Studies at LANL. A portion of this work was performed at the National High Magnetic Field Laboratory user facility, which is supported by the National Science Foundation Cooperative Agreement No. DMR-164479 the state of Florida and the U.S. Department of Energy.
[1] K. v. Klitzing, G. Dorda, and M. Pepper, Phys. Rev. Lett. 45, 494 (1980).

[2] M. König, S. Wiedmann, C. Brüne, A. Roth, H. Buhmann, L. W. Molenkamp, X.-L. Qi, and S.-C. Zhang, Science 318, 766 (2007).

[3] D. Hsieh, D. Qian, L. Wray, Y. Xia, Y. S. Hor, R. J. Cava, and M. Z. Hasan, Nature (London) 452, 970 (2008).

[4] M. Z. Hasan and C. L. Kane, Rev. Mod. Phys. 82, 3045 (2010).

[5] S.-Y. Xu, C. Liu, S. K. Kushwaha, R. Sankar, J. W. Krizan, I. Belopolski, M. Neupane, G. Bian, N. Alidoust, T.-R. Chang et al., Science 347, 294 (2015).

[6] C. Sürgers, G. Fischer, P. Winkel, and H. v. Löhneysen, Nat. Commun. 5, 3400 (2014).

[7] L. Ye, M. Kang, J. Liu, F. Von Cube, C. R. Wicker, T. Suzuki, C. Jozwiak, A. Bostwick, E. Rotenberg, D. C. Bell et al., Nature (London) 555, 638 (2018).

[8] E. Liu, Y. Sun, N. Kumar, L. Muechler, A. Sun, L. Jiao, S.Y. Yang, D. Liu, A. Liang, Q. Xu et al., Nat. Phys. 14, 1125 (2018).
[9] A. K. Nayak, J. E. Fischer, Y. Sun, B. Yan, J. Karel, A. C Komarek, C. Shekhar, N. Kumar, W. Schnelle, J. Kübler et al., Sci. Adv. 2, e1501870 (2016).

[10] S. Nakatsuji, N. Kiyohara, and T. Higo, Nature (London) 527, 212 (2015).

[11] N. Nagaosa, J. Sinova, S. Onoda, A. H. MacDonald, and N. P. Ong, Rev. Mod. Phys. 82, 1539 (2010).

[12] J.-X. Yin, S. S. Zhang, H. Li, K. Jiang, G. Chang, B. Zhang, B. Lian, C. Xiang, I. Belopolski, H. Zheng et al., Nature (London) 562, 91 (2018).

[13] E. Młyńczak, M. Eschbach, S. Borek, J. Minár, J. Braun, I. Aguilera, G. Bihlmayer, S. Döring, M. Gehlmann, P. Gospodarič et al., Phys. Rev. X 6, 041048 (2016).

[14] T. Suzuki, R. Chisnell, A. Devarakonda, Y.-T. Liu, W. Feng, D. Xiao, J. W. Lynn, and J. Checkelsky, Nat. Phys. 12, 1119 (2016).

[15] D. C. Tsui, H. L. Stormer, and A. C. Gossard, Phys. Rev. Lett. 48, 1559 (1982).

[16] R. B. Laughlin, Phys. Rev. Lett. 50, 1395 (1983). 
[17] W. Witczak-Krempa, G. Chen, Y. B. Kim, and L. Balents, Annu. Rev. Condens. Matter Phys. 5, 57 (2014).

[18] X.-G. Wen, Rev. Mod. Phys. 89, 041004 (2017).

[19] M. Dzero, K. Sun, V. Galitski, and P. Coleman, Phys. Rev. Lett. 104, 106408 (2010).

[20] H.-H. Lai, S. E. Grefe, S. Paschen, and Q. Si, Proc. Natl. Acad. Sci. USA 115, 93 (2018).

[21] K. R. Shirer, Y. Sun, M. D. Bachmann, C. Putzke, T. Helm, L. E. Winter, F. F. Balakirev, R. D. McDonald, J. G. Analytis, N. L. Nair et al., arXiv:1808.00403.

[22] M. Pasturel, O. Tougait, M. Potel, T. Roisnel, K. Wochowski, H. Noël, and R. Troć, J. Phys.: Condens. Matter 21, 125401 (2009).

[23] R. Troć, M. Pasturel, O. Tougait, A. P. Sazonov, A. Gukasov, C. Sułkowski, and H. Noël, Phys. Rev. B 85, 064412 (2012).

[24] See Supplemental Material at http://link.aps.org/supplemental/ 10.1103/PhysRevB.102.035127 for detailed theoretical model and experimental data.
[25] D. I. Gorbunov, I. Ishii, T. Nomura, M. S. Henriques, A. V. Andreev, M. Uhlarz, T. Suzuki, S. Zherlitsyn, and J. Wosnitza, Phys. Rev. B 99, 054413 (2019).

[26] D. I. Gorbunov, M. S. Henriques, A. V. Andreev, A. Gukasov, V. Petříček, N. V. Baranov, Y. Skourski, V. Eigner, M. Paukov, J. Prokleška et al., Phys. Rev. B 90, 094405 (2014).

[27] D. I. Gorbunov, T. Nomura, I. Ishii, M. S. Henriques, A. V. Andreev, M. Doerr, T. Stöter, T. Suzuki, S. Zherlitsyn, and J. Wosnitza, Phys. Rev. B 97, 184412 (2018).

[28] S. Onoda, N. Sugimoto, and N. Nagaosa, Phys. Rev. B 77, 165103 (2008).

[29] M. Lee, W. Kang, Y. Onose, Y. Tokura, and N. P. Ong, Phys. Rev. Lett. 102, 186601 (2009).

[30] M. Hirschberger, T. Nakajima, S. Gao, L. Peng, A. Kikkawa, T. Kurumaji, M. Kriener, Y. Yamasaki, H. Sagayama, H. Nakao et al., Nat. Commun. 10, 5831 (2019). 